\title{
Assessment of population radiation exposure at the edge of the exclusion zone 32 years after the Chernobyl accident: methods and preliminary results
}

\author{
J.-M. Bertho ${ }^{1,{ }^{*}}$, M. Maître ${ }^{2}$, P. Croüail ${ }^{2}$, W. Naito ${ }^{3}$, N. Shkliarava ${ }^{4}$, A. Mostovenko ${ }^{4}$, \\ K. Jones ${ }^{5}$ and M. Simon-Cornu ${ }^{6}$ \\ ${ }^{1}$ IRSN, PSE-SAN/SESANE, 31 avenue de la division Leclerc, BP 17, 92262 Fontenay-aux-roses, France. \\ ${ }^{2}$ CEPN, Fontenay-aux-roses, France. \\ 3 AIST, RISS, Tsukuba, Japan. \\ ${ }^{4}$ Research Institute of Radiology, Gomel, Belarus. \\ ${ }^{5}$ PHE, Chilton, UK. \\ ${ }^{6}$ IRSN, PSE-ENV/SEREN, Cadarache, France.
}

Received: 24 September 2019 / Accepted: 24 October 2019

\begin{abstract}
A case study was conducted in the village of Komarin in Belarus at the edge of the Chernobyl exclusion zone, with the aim of evaluating the spatial and temporal variability in external and internal postaccidental radiation exposure. A database containing the radioactive measurements of foodstuffs made in Komarin over a 1990-2016 period makes it possible to estimate retrospectively the internal exposure. External exposure was prospectively studied through a citizen science approach involving a group of high school students and used two radioactive monitoring approaches, the D-shuttle ${ }^{\odot}$ dosimeter and the OpenRadiation ${ }^{\circledR}$ system. Results showed that contamination level in food products varied over 2 to 4 orders of magnitude, underlining the usefulness of continuous monitoring of locally produced foodstuffs even more than 30 years after the Chernobyl accident. The study of external exposures showed that the current dose rates were similar to those usually observed elsewhere in the world, without any notable variations. More detailed analysis of these results will be conducted in future studies. This paper focuses on the methods used for the acquisition of data about both external exposure and foodstuff contamination and how it resulted in the strong involvement of high school students which in turn triggered a renewed interest in the practical radiological protection culture within the population of the village.
\end{abstract}

Keywords: Chernobyl / chronic exposure / practical radiation protection culture / citizen science

\section{Introduction}

The TERRITORIES European project was launched in January 2017 with the aim of reducing uncertainties and enhancing stakeholder involvement toward a graded approach for the management of exposure to ionizing radiation in long lasting exposure situations. Within this project, the workpackage \# 2 (WP2) was dedicated to identifying and assessing the factors which affect the uncertainty and variability of exposure by taking into account human behaviours. In this context, it was interesting to extend the investigations to a long-lasting post-accidental situation and especially to the study of the current radiological situation of a village at the edge of the Chernobyl exclusion area, in Belarus.

\footnotetext{
*Corresponding author: jean-marc.bertho@irsn.fr
}

The village of Komarin (1800 inhabitants, Bragin district, Gomel Oblast) was chosen because it is located along the river bank of the Dniepr River (the border between Belarus and Ukraine) a few kilometers east of the Chernobyl exclusion zone (Fig. 1). In 1986, many residents from surrounding villages were moved to Komarin after their houses had been dislocated and buried because of the high levels of radioactivity. The Komarin village was classified as being in the so-called "strict control area", meaning that the initial ${ }^{137} \mathrm{Cs}$ deposit in 1986 was estimated between $37 \mathrm{kBq}$. $\mathrm{m}^{-2}$ and $185 \mathrm{kBq} \cdot \mathrm{m}^{-2}$. Moreover, this official classification means that specific environmental and foodstuff survey has been ongoing for more than three decades to ensure that the exposure of the population remains acceptable with respect to the regulatory limits as set by authorities (Bataille and Croüail, 2005). 
A

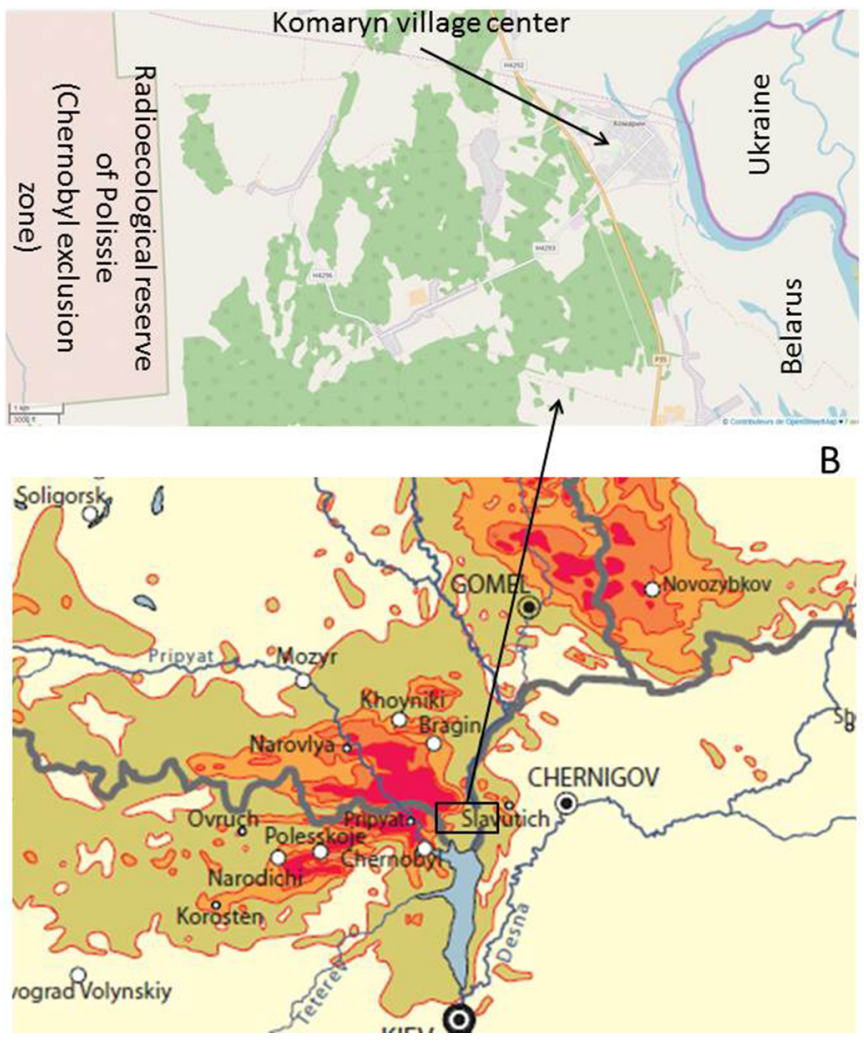

Fig. 1. A. Location of the Komarin village. The map is extracted from the Openstreetmap web site (www.openstreetmap.org). B. ${ }^{137} \mathrm{Cs}$ contamination map of the Chernobyl area as defined for year 1986 after fallout deposition. The map is extracted from UNSCEAR report (UNSCEAR, 2011) with courtesy.

The human exposure to artificial radioactivity in a postaccidental situation is composed of two components: the internal exposure due to the ingestion of contaminated foodstuffs due to the presence of radionuclides in agricultural products (including products from animals contaminated by feedstuffs) and the external exposure due to the presence of radionuclides in the environment. These two components vary according to the time after deposition, as well as their respective proportion in total exposure (Bernhardsson et al., 2011). The first one, internal exposure, was approached by collecting the radioactive measurement data of foodstuffs and feedstuffs that were made by various official organisations and were gathered into a database covering the 1990-2016 period. The second component was evaluated using two different measurement systems, the OpenRadiation ${ }^{\circledR}$ kit (BottollierDepois et al., 2017; Bottollier-Depois et al., 2019) for the measurement of the ambient dose rate and the D-shuttle ${ }^{\odot}$ dosimeter (Hirayama, 2013) for the measurement of individual external doses. Since we hypothesized that a modification of the individual behavior depends primarily on the knowledge of the radiological situation, as it was firstly proposed with the development of the concept of the practical radiation protection culture during the ETHOS European project (Dubreuil et al., 1999; Lochard, 2007), it was decided to adopt a citizen science approach involving the teachers and students of the secondary school of Komarin in these measurements. Such collaborative approach with citizen has been shown efficient in improving radiation protection of concerned populations (Liland and Skuterud, 2013). In fact, the citizen science in radiation protection has been reemphasized in the aftermath of the accident of the Fukushima Daiichi Nuclear Power Plant as a powerful tool to acquire useful and reliable data for further assessments (Brown et al., 2016) and to engage concerned populations to be participants in their radiation protection (Reiher, 2016; Miyazaki, 2017; Ando, 2018; Lochard et al., 2019), and this is in line with the radiation protection principles and ethics developed by ICRP $(2009,2018)$. Overall, this case study is expected to provide a complete picture of the radiological situation of a whole village and to give information about the perception and attitudes toward radioactive contamination by the population in the long-term aftermath of a major nuclear accident. The work presented here gives an overview of the methods used to reach these goals and a preliminary view of the results obtained.

\section{Material and methods}

\subsection{Foodstuff and feedstuff measurement data collection and creation of the database}

The foodstuff and feedstuff measurements were carried out mainly by three Belarusian organizations, the veterinary station in Komarin (Vet St), the Bragin district sanitary and epidemiology station (SES) and the Komarin local center for the promotion of the radiation protection culture (CPRC). Each of these organizations used various devices during the study period (1990-2016) with different levels of sensitivity (Tab. 1). The detection limit (DL) of the instruments has decreased significantly in recent years, due to the increased quality of the detectors used. All measurement instruments were regularly controlled and calibrated for cesium-134 and cesium-137 detection by the organizations which were accredited by the Belarusian State Committee for Standardization, Metrology and Certification.

The measurements were made according to a classical protocol, consisting of weighting the product as it arrives in the lab (minimal weight of $500 \mathrm{~g}$ ) before measuring the total activity of the sample. In fact, products were usually provided as fresh products, i.e., wet in most cases except for hay and some mushroom samples that were essentially dried by the producer. Results were expressed in $\mathrm{Bq}$ per $\mathrm{kg}$ of the product as received by the lab (i.e., of fresh weight in most cases). During the early period (until 2002), most of the data were collected in notebooks and were later computerized. All this data were gathered in a single Excel file and checked for the coherence of the units used and the values recorded. Data originally recorded in $\mu \mathrm{Ci} \cdot \mathrm{kg}^{-1}$ were converted to $\mathrm{Bq} \cdot \mathrm{kg}^{-1}$. Measurement uncertainties were not evaluated and values indicated as below the DL are ascribed the DL value.

It should be noted that some of the data in the form of notebooks were irretrievably lost because of different external events (flooding, fire, rodents, etc.), explaining the shortage of data for the 1990-1995 period and some other years. 
Table 1. List of radiometers used in the three institutions in charge of radiation control and associated detection limits. All radiometers are provided by Atomtex (Ukraine).

\begin{tabular}{|c|c|c|c|}
\hline Institution & Period & Model & Detection limit, ${ }^{137} \mathrm{Cs}$ (Bq.kg $\left.{ }^{-1}\right)$ \\
\hline \multirow[t]{2}{*}{ Center for the practical radiation protection culture (CPRC) } & $\leq 2001$ & RUG 92 & 18 \\
\hline & 2002-2004 & RUG 92M & 11 \\
\hline \multirow[t]{3}{*}{ Bragin district sanitary and epidemiology station (SES) } & $1986-1991$ & KRVP-3AB & 185 \\
\hline & 1991-1997 & RKG-05P & 20 \\
\hline & 1997-2001 & RKG-01A/1 & 7.4 \\
\hline \multirow[t]{3}{*}{ Komarin veterinary station (Vet St) } & 1994-1996 & KRVP-3AB & 185 \\
\hline & $1997-2013$ & RKG-92 & 18 \\
\hline & $\geq 2014$ & RKG-AT1320 & 3.7 \\
\hline
\end{tabular}

\subsection{Historical ambient dose rate measurements and whole body counting (WBC)}

Other historical measurements were also included in the database. Ambient dose rate, both indoor and outdoor, were measured under the responsibility of the Research Institute of Radiology (RIR) at the official reference point few kilometers away from the village. Unfortunately, no details are given about the nature of the reference building and the radiometer used for these measurements. Whole body counting (WBC) were made under the responsibility of two different institutes, the Belrad institute and the regional center for hygiene and epidemiology (RCHE), using seat-type whole body counter. WBCs were available for school children for years 1995,2000 , 2004-2007, 2010, 2015 and 2016. Each child was ascribed a unique identifying number with age, sex and body weight registered at the time of measurement.

\subsection{OpenRadiation ${ }^{\circledR}$ measurements}

In order to evaluate the current ambient dose rate in the village an action was conducted using the OpenRadiation ${ }^{\circledR}$ system (www.openradiation.org) (Bottollier-Depois et al., 2017; Bottollier-Depois et al., 2019). The system is composed of a kit with a gas detector controlled through Bluetooth communication by an application on a smartphone (www. openradiation.org). The application is set so that measurements are made with at least 50 counts registered, providing dose rate measurement uncertainty less than $15 \%$. The minimal duration of dose rate measurement is thus dependent on the dose rate at the place of measurement. However, participants have the choice to measure for longer duration. As a result, the mean number of counts per measurement is $54 \pm 16$ (range: $50-348$, $n=634$ ) with $82.6 \%$ of measurements obtained with less than 55 counts. The measurements are then described by tags chosen by the user regarding the location of the measurement (possible choices: indoor/in the countryside/on the road/in the city), the weather (sunny/rainy) and the height above the ground of the detector at time of measurement (on the ground/ one meter above the ground). Then the measurement is sent with associated metadata (date, time and GPS location of the measurement, data regarding the detector, and data regarding the smartphone and the application) to the website and is immediately visible on the interactive map of the website.
Each measurement is associated with the username of the person who performed the measurement.

A group of 17 students from the Komarin School, aged 1417 years old, were recruited on a voluntary basis to participate in the OpenRadiation study. The following minimal instructions were given to the participants:

- measurements can be made at any time and any place, at the choice of the participant, with the exception of forbidden areas, such as workspace, private properties, already known highly contaminated areas, Chernobyl exclusion zone. As a whole, the participants were prompted to avoid taking any risk;

- if "hotspots" are identified, then avoid staying too long in these places;

- any measurement should be identified by the username of the participant and described by the proposed tags before sending it to the website.

A supplementary recommendation was to take measurements at about one meter above the ground but this was not mandatory because it does not significantly change the dose rate for a homogeneous soil contamination. Whatever the choice of the participant, it was asked that all measurement were tagged for the position of the detector above the ground. After one month, measurements were stopped and results were downloaded from the OpenRadiation website. The data were then extracted from the OpenRadiation website based on the username of the students.

\subsection{D-shuttle $\subset$ dosimetric measurements}

This study was made possible thanks to the National Institute of Advanced Industrial Science and Technology (AIST, Japan), that provided $20 \mathrm{D}$-shuttle ${ }^{\odot}$ and the associated system for instant reading of daily and total dose. D-shuttle ${ }^{\odot}$, which is manufactured by Chiyoda Technology Corp. (http:// www.c-technol.co.jp/eng/e-dshuttle), measures personal dose equivalent $[\mathrm{Hp} 10]$. Since D-shuttle ${ }^{\odot}$ measurements under the conditions encountered in the affected areas such as Fukushima were comparable with the effective dose of isotropic (ISO) or rotational (ROT) irradiation geometries (Hirayama, 2013), the individual dose measured by D-shuttle ${ }^{(}$ is considered as a realistic indicator of the effective dose from 
external radiation exposure. Several municipalities in the affected areas in Fukushima provided a D-shuttle ${ }^{\circledR}$ to each resident to measure and help them understand their individual external dose.

A group of 19 participants, including the 17 students who used the OpenRadiation ${ }^{\circledR}$ detector, wore the D-shuttle ${ }^{(}$and were then asked to fill a diary sheet to describe their daily activities as well as the time spent and the location (indoor/ outdoor) of these activities. These D-shuttle ${ }^{\circledR}$ were sent back to Japan for analysis. Out of $19 \mathrm{D}$-shuttle ${ }^{\circledR}$ provided, 19 were worn for two consecutive weeks by 19 different participants with a fulfilled diary and were used for analysis.

\subsection{Survey of participants to the study}

At the end of the Komarin on-site study, a meeting was organized with members of the TERRITORIES project, students who performed measurements, parents and the villagers interested by the project. This meeting aimed at presenting the results of the OpenRadiation ${ }^{\odot}$ and the DShuttle ${ }^{\odot}$ studies to discuss them with the participants and to collect their views and expectations for the future. During this meeting, a survey was also proposed to the students and to the meeting participants. This survey was divided in four parts. The first part included personal data, the second and the third parts were related to the ergonomics of the OpenRadiation ${ }^{\circledR}$ system and the way the students organized their measurements (these two parts were specific to the questionnaire for students), and the last part concerned the feelings of the participants about the possibility of making ambient dose rate measurements by themselves. Fifteen questionnaires from students and 25 questionnaires from villagers were completed and used for subsequent analysis.

\subsection{Ethical issues and protection of personal data}

WBC data that were provided for the study had already been anonymized. All participants of the project were asked to sign an informed consent for the use of their personal information regarding the D-shuttle ${ }^{\circledR}$ and the OpenRadiation ${ }^{\circledR}$ studies. Data on the OpenRadiation ${ }^{\circledR}$ website were anonymized through the use of a username. As well, questionnaires filled out by adults during the restitution meeting were anonymized. All participants gained the right to access and to modify their personal information through direct contact with the project managers.

\subsection{Statistical analyses}

Unless otherwise indicated, results are presented as mean \pm standard deviation (SD), or as median, 10th, 25th, 75th and 90th percentiles and ranges. Group comparisons were made using one way, two ways or on ranks ANOVA tests, as indicated in the text. Statistical analyses were made using Sigmaplot statistical software (V11.1, Systat, Paris, France). Differences were considered statistically significant for $p<0.05$.

\section{Results}

11000 lines of data were registered in the database, covering a period from 1990 to 2016.

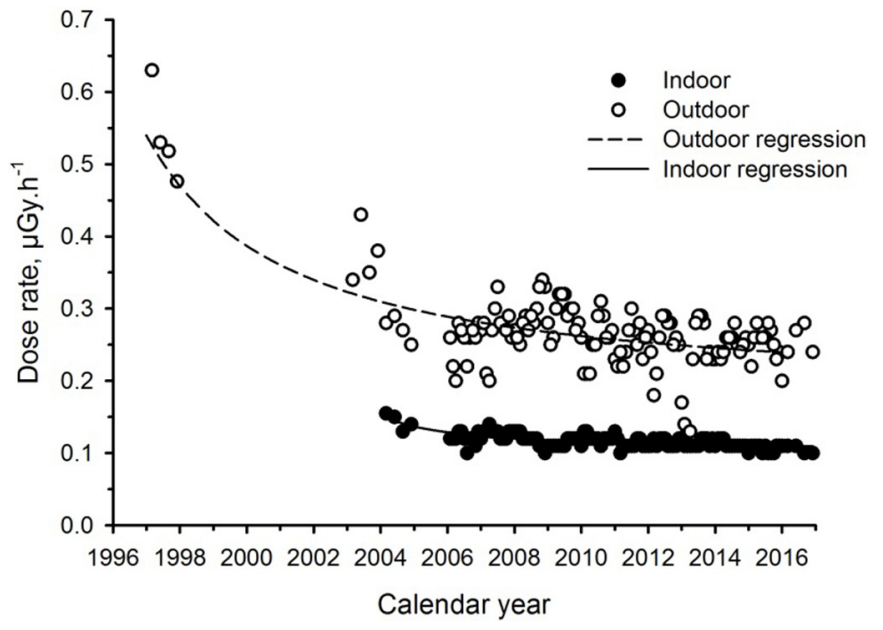

Fig. 2. Ambient dose rate measured at the official reference point in a building at a few $\mathrm{km}$ distance from the village from 1997 to 2016. Each dot represents the monthly or the quarterly mean dose rate. Closed circles: indoor ambient dose rate; open circle: outdoor dose rate. The lines represent the non-linear regression of each dataset. Adjusted regression squares are, $r^{2}=0.4759$ for Indoor data and $r^{2}=0.8967$ for outdoor data. The apparent half-life for outdoor dose rate is 18.15 years.

\subsection{Ambient dose rate results}

Ambient dose rates constitute a first group of results in the database. They were measured at an official reference point located few kilometers outside the village. The measurements were made both indoor and outdoor and provided a complete time series from 2004 to 2016 for indoor dose rates and from 1997 to 2016 for outdoor dose rates, with a gap between 1998 and 2002 (Fig. 2). A consistent decrease in ambient dose rates is observed during the period. The non-linear regression analysis based on the hypothesis of an exponential decay yield a $\mathrm{r}^{2}=0.476$ for indoor dose rate and $\mathrm{r}^{2}=0.897$ for outdoor data with an apparent half-life of 18.15 years (Fig. 2). The indoor ambient dose rate is consistently lower that the outdoor dose rate. For the years 2015 and 2016, the mean ambient dose rates are $0.107 \pm 0.005 \mu \mathrm{Gy} \cdot \mathrm{h}^{-1}$ and $0.107 \pm 0.006 \mu \mathrm{Gy} \cdot \mathrm{h}^{-1}$ indoor and $0.251 \pm 0.024 \mu \mathrm{Gy} \cdot \mathrm{h}^{-1}$ and $0.263 \pm 0.021 \mu \mathrm{Gy} \cdot \mathrm{h}^{-1}$ outdoor, respectively. This indicates an indoor shielding factor in the reference building of 0.43 and 0.39 in 2015 and 2016 respectively.

\subsection{Foodstuff and feedstuff radioactive measurements}

The second group of data consists of foodstuff and feedstuff measurements covering also a period from 1990 to 2016. Each line of data includes the date of measurement, the name and the status (private, industrial or commercial) of the provider, the nature of the foodstuff or feedstuff sample, and the resulting activity in $\mathrm{Bq} \cdot \mathrm{kg}^{-1}$ of wet weight, except for some products such as hay and mushrooms which were measured as received, i.e., partly dried by the provider. For some periods, the address of the provider was also indicated, allowing a more precise location of the private garden. Given 


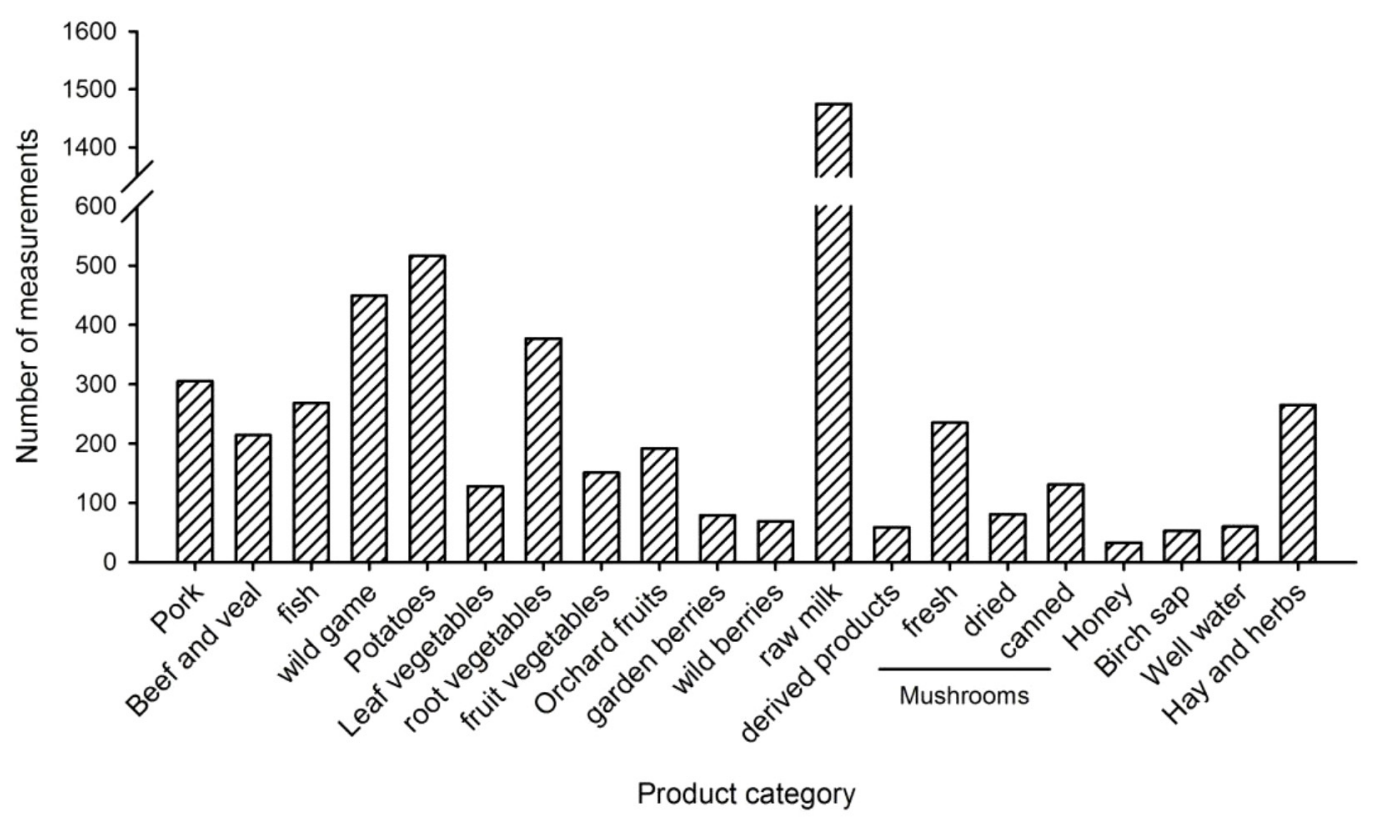

Fig. 3. Number of measurements in the database for each of the 20 categories of foodstuff or feedstuff for year 2015 .

the high number of different products measured along the period covered by the database, products were grouped into 20 different categories. Figure 3 gives the number of measurements in each of these 20 categories. Since the measured products were brought mostly by individuals on a voluntary basis ( $85 \%$ of the measurements, ranging from $48.6-$ $100 \%$ according to the product category), the proportion of the different products is considered to provide a realistic picture of the type of food used in the diet of Komarin residents. The milk appears as a major food item in the local diet (1475 measurements), as well as garden vegetables (656 measurements for the three vegetable categories), potatoes (516 measurements) and wild game (449 measurements). This part of the database represents approximately 5200 lines of data over the whole period of the study.

As an example, Figure 4 shows the results of measurements of foodstuffs for the year 2015. As can be seen, most of the measured products are well below the Republican Permissible Levels (RPL, see Tab. 2) set by the Belarusian authorities, which is currently $80 \mathrm{~Bq} \cdot \mathrm{kg}^{-1}$ for the potatoes for instance. However, some samples show a ${ }^{137} \mathrm{Cs}$ contents very close to the RPL, such as one potato sample $\left(76.2 \mathrm{~Bq} \cdot \mathrm{kg}^{-1}\right)$, underlying the importance of foodstuff monitoring even more than 30 years after the Chernobyl accident. The most contaminated products are hay and pasture samples (used for livestock feeding) and mushrooms harvested in the vicinity of the village. In our database, hay and mushrooms are consistently the two most contaminated products all over the years.

An interesting feature of this database is the long period over which measurements were made. It allows observing the temporal evolution of each individual food item. An example is given in Figure 5, with the evolution of the contamination of potatoes during the period 1990-2016. It shows that the observed variations are not simply explained by the radioactive and environmental decay. Different hypotheses may explain such variations, such as a bias in the sampling, the influence of river flood or the differential use of potassium fertilizers along the period of the study. Other products, not directly under human cultivation, such as wild game and mushrooms, showed a continuous decrease in the level of contamination, although large variability within a single year was evident.

\subsection{Ambient dose rate map of the village}

Three OpenRadiation ${ }^{\circledR}$ measurement kits were provided to the group of 17 students of the Komarin School at the beginning of June 2018, for a period of one month. Demographic characteristics of the participants are described in Table 3. They were accompanied by the physics teacher who gave special courses on radioactivity and basic principles of radiation protection. Each participant was thus able to use the measurement kit for a period of 3 to 5 consecutive days and to understand the meaning of the results. A total of 645 measurements were made during this one month period. Altogether they provided a very comprehensive radiological map of the village (Fig. 6). Overall, the dose rates observed in the village were close to those usually observed elsewhere in the world (UNSCEAR, 2008), including France (IRSN, 2016) although some variations in the range of $0.050-0.950 \mu \mathrm{Sv}_{\mathrm{h}}{ }^{-1}$ can be observed. This confirm a previous study using the D-shuttle ${ }^{\odot}$ showing that individual external doses measured in and outside the Fukushima prefecture (Japan), in Poland, in Belarus (Gomel and Bragin towns) and in France (Poitiers, Bastia and Boulogne towns) were in the same order of magnitude (Adachi et al., 2016). Since all measurements were tagged with one out of four possible locations (namely "inside", "in the countryside", "in the city" and "on the road") and are GPS-located, a more detailed analysis of these ambient dose rate according numerous parameters will be done. For instance, "inside" measurements are significantly higher than other measurements tagged "countryside", "on the road" or "in the city" 

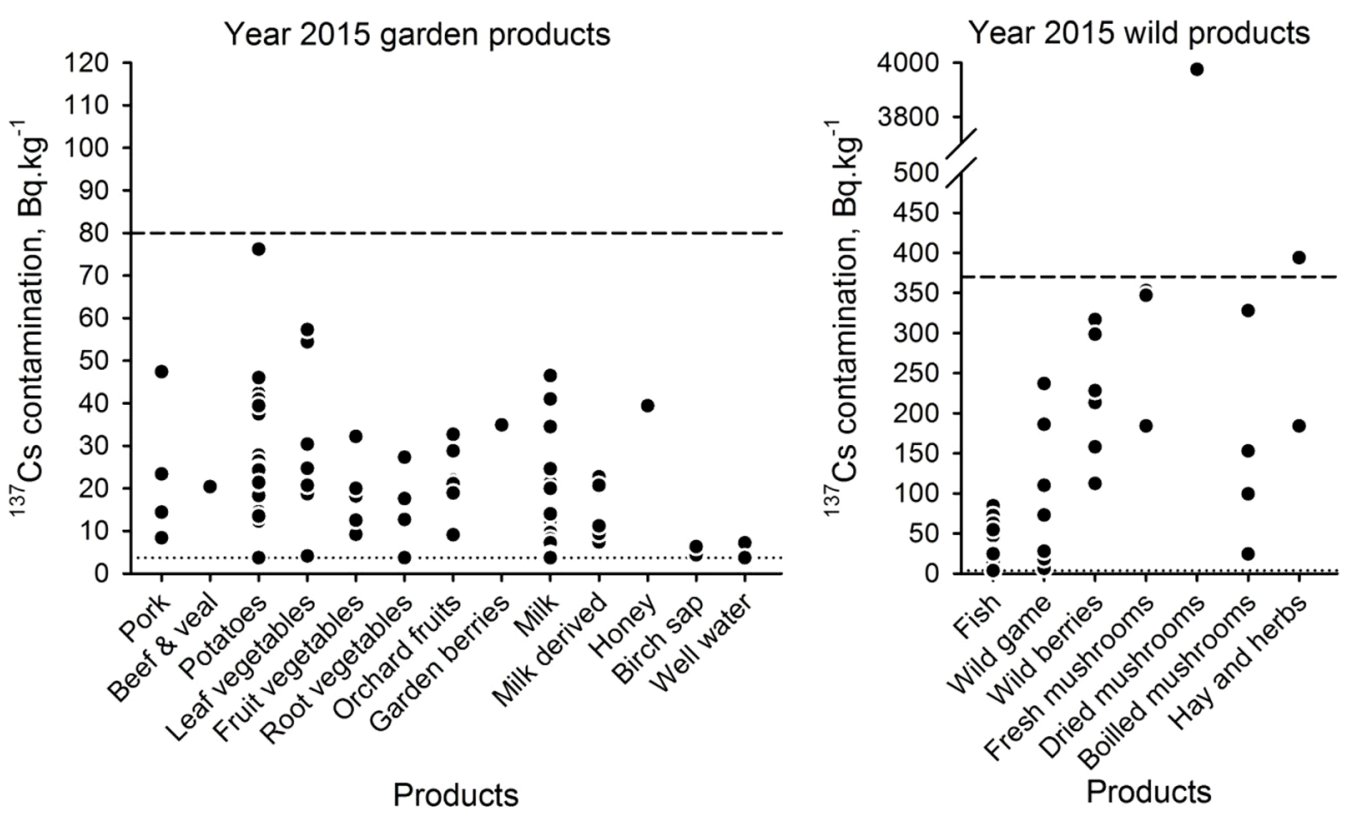

Fig. 4. Contamination level of foodstuffs grouped in 20 categories as measured during year 2015. The horizontal dashed line corresponds on the left to the RPL 99 for potatoes and on the right to the RPL 99 for fresh mushrooms. The horizontal dotted line represents the detection limit for ${ }^{137} \mathrm{Cs}$ (see Tab. 1).

Table 2. Established limits of ${ }^{137} \mathrm{Cs}$ concentrations in foods (Bq.kg ${ }^{-1}$ or Bq. $\left.\mathrm{L}^{-1}\right)$ at different periods of time after the Chernobyl accident in Belarus (republican permissible levels).

\begin{tabular}{lccc}
\hline Food product & TPLs-88 & RPLs-92 & RPLs-99 \\
\hline Drinking water & 18.5 & 18.5 & 10.0 \\
Milk & 370 & 111 & 100 \\
Beef, mutton, sausage products & 2960 & 600 & 500 \\
Pork, poultry, fish, eggs & 1850 & 600 & 180 \\
Potatoes & 740 & 370 & 80 \\
Vegetables & 740 & 185 & 100 \\
Fruits & 740 & 185 & 40 \\
Bread, bakery products, pasta & 370 & 185 & 40 \\
Dried mushrooms & 11100 & 3700 & 2500 \\
Wild berries & & & 185 \\
Wild game & & & 500 \\
Fresh mushrooms & & & 370 \\
\hline
\end{tabular}

a Temporary permissible levels set in 1988 .

b Republican permissible levels set in 1992.

${ }^{c}$ Republican permissible levels established in 1999 and still in effect at the present time.

(Fig. 7). A more detailed analysis of these data will be provided in a future study.

Overall, the high number of measurements made by the students in a short period of time and with a limited number of OpenRadiation ${ }^{\circledR}$ tools confirms the interest of post-millennials for the citizen science approach using modern and easy-to-use equipment and apps. The creation by themselves of such a radiological map of the village allowed them to gain the knowledge of the radiological situation of their living place and to reconsider their environment.

\subsection{D-shuttle ${ }^{\circledR}$ measurements}

$19 \mathrm{D}$-shuttle ${ }^{\odot} \mathrm{s}$ were worn by the participants and associated diaries fulfilled with enough details so that these data will be useable for a future analysis. For each participant, a complete profile was obtained, showing no notable variations in the hourly dose (Fig. 8A). As observed with the OpenRadiation ${ }^{\circledR}$ measurements, the overall hourly dose for each participant is within a range of $0.07 \mu \mathrm{Sv} / \mathrm{h}$ to $0.12 \mu \mathrm{Sv} / \mathrm{h}$ (Fig. 8B). This is comparable to external exposure measured elsewhere in the world, as described in a previous study (Adachi et al., 2016). This corresponds to an annual external background exposure in the range of $0.6-1.1 \mathrm{mSv} / \mathrm{year}$, comparable to the external annual exposure in France for instance (IRSN, 2016) or elsewhere in the world (UNSCEAR, 2008).

\subsection{Survey of the participants to the study}

All the data obtained during this study were presented to the participants and to the villagers during a meeting in the village. At this occasion, all the participants stressed their interest to be involved in this study as it has been a real opportunity for them to reconsider their environment, to remember the past of their territory as well as to testify their own experiences. Moreover, participants also showed a strong interest in the study results and even sought to compare these results with their daily practices. For instance, the use of wooden ashes as a fertilizer in vegetable gardens has been mentioned. Some residents confirmed that this is still a common practice despite recommendations provided by 


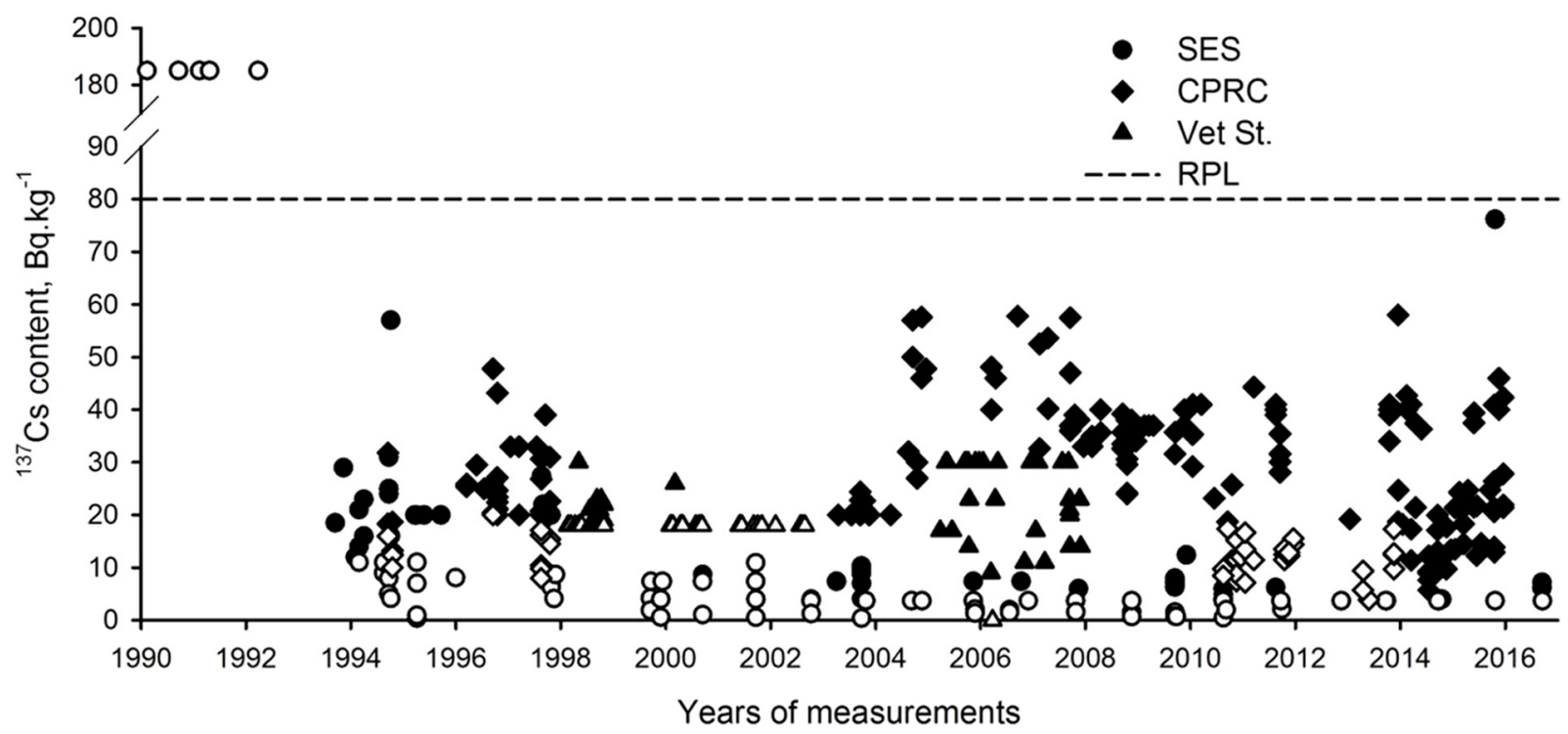

Fig. 5. Evolution of potato contamination level $\left(\mathrm{Bq} \cdot \mathrm{kg}^{-1}\right)$ during the study period. Data were collected from three different institutions namely the sanitary and epidemiology station (SES, circles), the center for the promotion of radiation protection culture (CPRC, diamonds) and the veterinary station (Vet st, triangles). The horizontal dashed line represents the RPL99 level for potatoes (see Tab. 2) and the open symbols indicate measurements at or under the detection limit (see Tab. 1).

Table 3. Demographic description of the 17 participants from the Komarin village to the OpenRadiation ${ }^{\circledR}$ project.

\begin{tabular}{llr}
\hline Parameter & Number $^{\mathrm{a}}$ & Percent \\
\hline Participants & 17 & 100.0 \\
Male & $9 / 17$ & 52.9 \\
Female & $8 / 17$ & 47.1 \\
Age class & & \\
14-15 & $3 / 16$ & 18.8 \\
15-16 & $7 / 16$ & 43.7 \\
16-17 & $6 / 16$ & 37.5 \\
Vegetable garden & & \\
Yes & $11 / 14$ & 78.6 \\
No & $3 / 14$ & 21.6 \\
Nature of housing & & \\
Collective & $6 / 15$ & 40.0 \\
Individual & $9 / 15$ & 60.0 \\
Main building material & & 33.3 \\
Wood & $5 / 15$ & 26.7 \\
Bricks & $4 / 15$ & 40.0 \\
Cinder block/concrete & $6 / 15$ & \\
\hline
\end{tabular}

a The amount of available information varies depending on questionnaire contents. In several cases, answers to some questions were missing. Percentages were then calculated according to the total number of available answers.

authorities in the past not to do so. Therefore, these particular issues have been investigated and taken into consideration by local authorities by providing appropriate information and safety reminders to the residents.

The meeting in the village was also the occasion for asking the participants to fill out a questionnaire about their feelings and thoughts about the present study. The motivation to participate in the study was mainly for the curiosity $(60 \%)$, then for the knowledge about the areas with high dose rate $(20 \%)$ and for protecting themselves and their family $(15 \%)$. Results showed that the students have chosen to measure first their home (70\%) which was confirmed by the GPS location of the measurements. Thereafter, they choose to measure the vegetable garden (34\%), some public places (park, stadium, riverbank, $40 \%)$ and their usual paths in the village $(13 \%)$. Interestingly, $80 \%$ of the participants shared and discussed the results of their measurements with their teachers, family or friends, and $60 \%$ compared their measurements with the results of the OpenRadiation ${ }^{\circledR}$ community through the website. All of the students asked for a dissemination of their results not only through the web site but also through other communication means such as newspapers or information booklets. Again, all of these results demonstrate the strong interest of the students in participating to the study, for the knowledge of the radiological situation of the village and for its dissemination to increase awareness of residents and their vigilance. This is further demonstrated by the fact that $80 \%$ of the students wanted to continue taking measurements with $60 \%$ of them with an OpenRadiation kit at disposal.

\section{Discussion}

This study combined an evaluation of external exposure through a citizen science approach using two different devices (the OpenRadiation ${ }^{\circledR}$ kit and the D-Shuttle ${ }^{\circledR}$ dosimeter) together with a study of the level of contamination in locally produced foodstuffs, over a long period of time ( 25 years) after the major nuclear accident of Chernobyl. Such an approach at the scale of a single village allows for the assessment of exposure in a reliable way even if individual dose reconstruction would remain difficult. The large variation in 


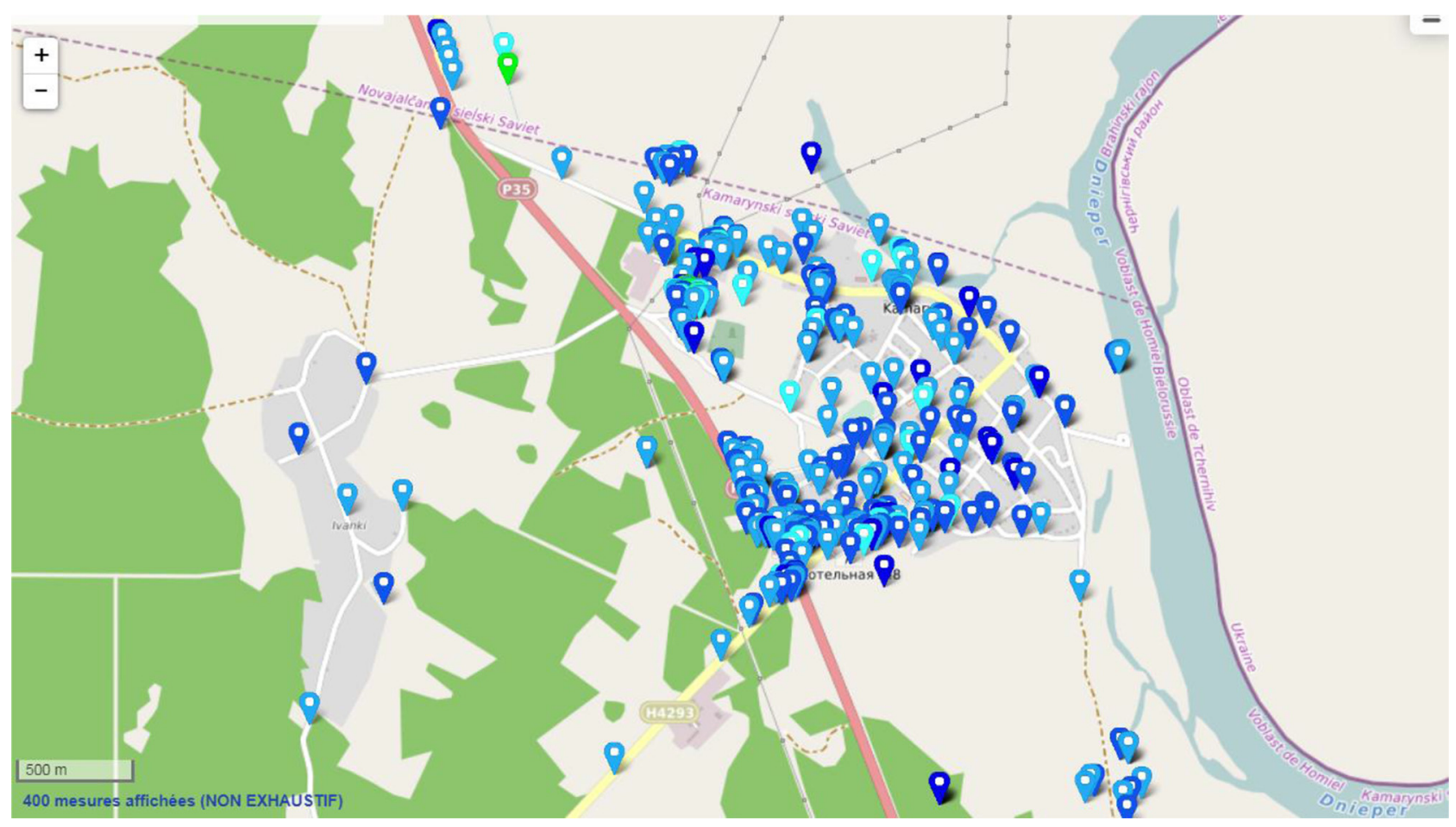

Fig. 6. Map of measurements made by the group of 17 students from the Komarin school. Each dot represents a single measurement, for a total of 645 measurements made during June 2018. Only 400 measurements are displayed simultaneously on the map of the website. The color code indicates the level of ambient dose rate from the lowest (dark blue) to the highest (green). The map is extracted from the OpenRadiation ${ }^{\circledR}$ web site.

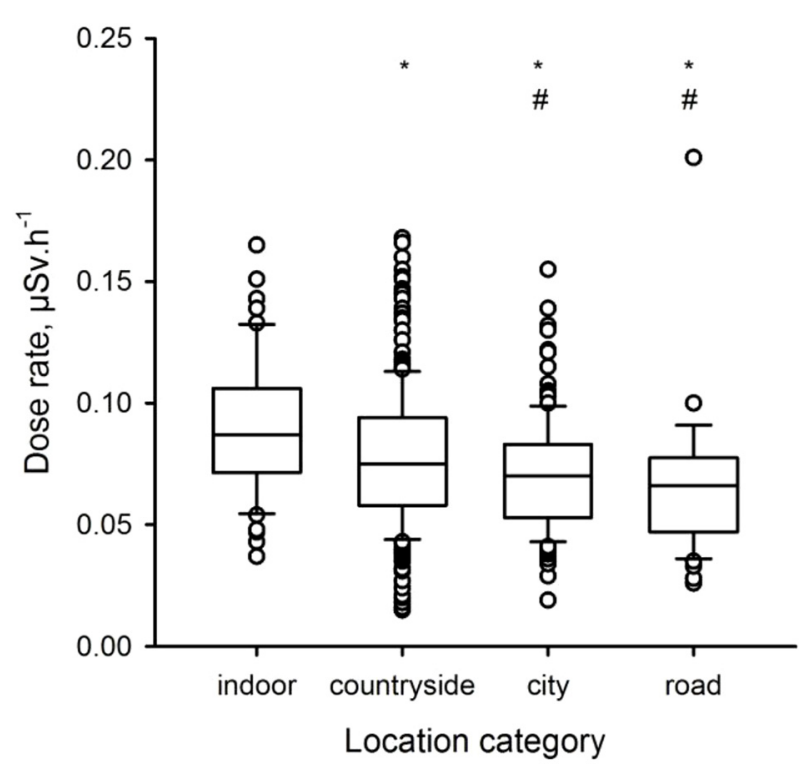

Fig. 7. Ambient dose rates measured with the OpenRadiation ${ }^{\circledR}$ kit according to the tag used to describe the location of the measurement. Bars and boxes indicate the 10th, 25th, median, 75th and 90th percentiles. Differences between groups are significantly different for $p<0.05$ as compared to indoor tag $\left(^{*}\right)$ and to countryside tag (\#).

the contamination level of foodstuff, over 2 to 4 order of magnitude depending on the products and on the calendar year suggests that some variations should be also observed in the evaluation of human exposure. It should be noted that the level of locally produced foodstuffs are in most cases below the RPLs, while products harvested from the environment may frequently exceed them. One has to note that the current Belarusian regulation (RPL 99) was set to limit the exposure due to foodstuff contamination to $1 \mathrm{mSv}$ per year, which correspond to a daily ingestion of $185 \mathrm{~Bq}{ }^{137} \mathrm{Cs}$ per day, on the basis of the mean Belarusian diet (Bataille and Croüail, 2005). Such variability in foodstuff contamination suggests that food monitoring should be carried on even more than 30 years after the Chernobyl accident.

In fact, the organization of radiation protection in the Komarin village has been mainly centered on two different actions. The first one was the measurement of food contamination offered to the population, on a volunteer basis. This was achieved thanks to the presence of the "radiometrist" of the village who was in charge of this monitoring since 1986. The second action was based on the existing education program in Belarus about radiation protection. This education was provided either during the school time or in the context of specific radiation protection club, aiming at disseminating a radiation protection culture within the population of the village. The part of the present study using the D-shuttle ${ }^{\odot}$ and OpenRadiation $^{\odot}$ tools allowed to measure the real levels of ambient radiation dose rates within the Komarin village, that varied slightly but remains in the usual range as compared to other places in the world (Adachi et al., 2016; IRSN, 2016; UNSCEAR, 2008). However, ambient dose rate measured at the official reference point showed higher ambient dose rates, both indoor and outdoor. This is clearly due to the location of the reference point, located at the entrance of the Chernobyl exclusion zone. As a result, dose rates measured at this 

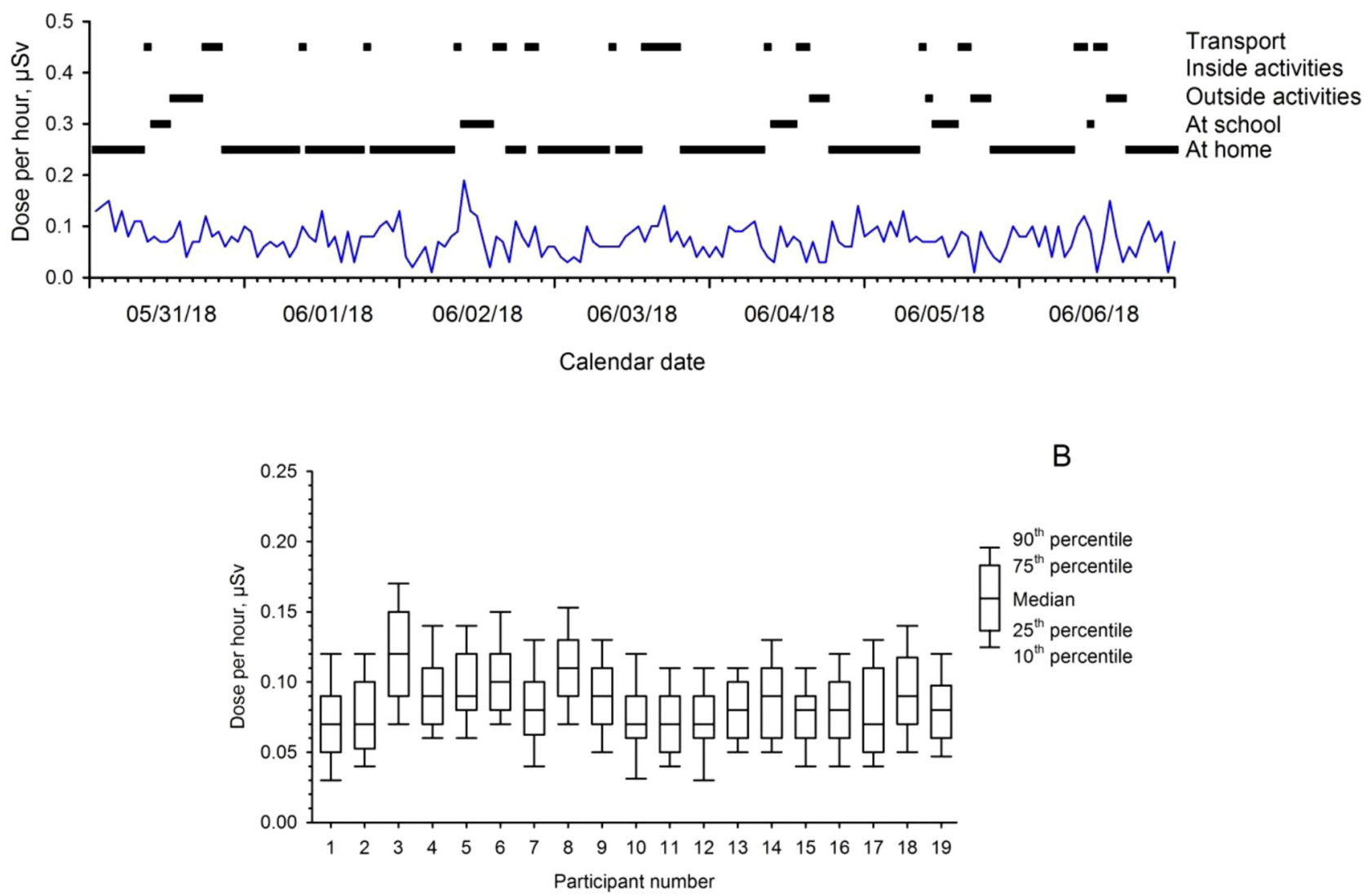

Fig. 8. Results of the D-Shuttle ${ }^{\odot}$ study. A. Dosimetric profile of a participant over one week. The blue line indicates the dose per hour and the horizontal colored lines indicate the activity according to the diary. B. Median dose per hour with 10th, 25th, 75th and 90th percentiles for each of the 19 participant.

reference point are not representative of the ambient dose rate in the Komarin village. Nevertheless, three "hot spots"

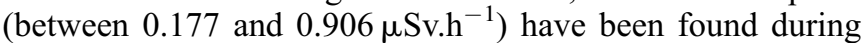
the OpenRadiation ${ }^{\circledR}$ measurements in non-residential areas. These "hot spots" corresponded to ash storages. This highlighted the necessity to keep monitoring ambient dose rates in the village, and from time to time to remind the population practical tips and advices for better radiation protection.

The citizen science approach used in this part of the study has been very successful from the point of view of stakeholder engagement and to highlight radiation protection culture, as demonstrated by the 645 measurements made by the group of students with only three measurement kits and in a very short period of time. The reasons of this success might be multiple, with two main reasons to be pointed out. Firstly, the presence of radioactivity in the environment is made visible only through measurements (that is, "make visible the invisible"). Secondly, the real-time nature of the result, visible on both the smartphone app and on the OpenRadiation ${ }^{\circledR}$ map was of great interest to the participants. Moreover, this allowed the participants to make comparisons of the local level of ambient dose rate with other places word-wide and consequently to balance and to contextualize the risk they are facing. These conclusions are fully supported by the discussions during the final meeting as well as the results of the questionnaire fulfilled by the participants. Finally, there was a strong demand made by the children and their parents that such ambient dose rate measurements should be continued in the village. They argued in favor of making the measurement themselves so that they could measure "what they want, where they want and when they want". This is probably stressed by the unexpected discovery of three hotspots in the village, highlighting that local concentrations of radioactivity may arise. This called for, from time to time, a redefinition of protective measures and radiation safety information to be provided to the residents.

However, the dataset obtained using the OpenRadiation ${ }^{(}$ might be biased for several reasons, including wrong tagging of the measurement, measurements made at variable heights above the ground, choices of place of measurements biased by the personal interest of the individual for instance. Thus, the quality of the dataset might be difficult to assess. However, whereas some individual measurements may be questionable, the whole dataset as a mean is probably representative of the real radiological condition. On the other hand, it is reasonable to suggest that since the students were monitoring their own living environment for health-related matters the measurements were done seriously with some accuracy. This is supported by the fact that there was a low proportion of measurements with missing information (lack of a tag for 
instance). Besides, this is further supported by results of previous studies, showing that crowd sourced measurements were in good agreement with official measurements in the Fukushima prefecture (Nursal et al., 2016; Hultquist and Cervone, 2018).

In term of future work, the comprehensive data collection will allow studying the spatial and the temporal evolution of both internal and external body exposures. More specific analyses will be conducted based on the collected data. A detailed study regarding the level of ${ }^{137} \mathrm{Cs}$ ingestion, based on a mean alimentary survey, will allow the evaluation of variability in internal exposure according to the calendar year. Such a study will also allow the evaluation of the relative contribution of external $v s$. internal exposures across calendar years. Lastly, results of such a study could be confirmed by comparison with WBC measurement results, also included in the database (not presented here). Other future studies could be conducted on the basis of data collected with the D-shuttle ${ }^{\odot}$, with the GPS-localized OpenRadiation ${ }^{\odot}$ measurements and with the activity diaries written by the students. For example, the preliminary analysis of OpenRadiation ${ }^{\odot}$ data showed that mean indoor ambient dose rates are higher than mean outdoor ambient dose rates (see Fig. 7), which is unusual and in contradiction with the measurements at the reference point (Fig. 2). A more detailed study about the indoor/outdoor ambient dose rate ratio will be conducted using the data obtained in the OpenRadiation ${ }^{\circledR}$ and the D-shuttle ${ }^{\odot}$ studies, together with an analysis of the time spent indoor (indoor occupancy budget). These two parameters are important when evaluating the exposure of populations in emergency or post accidental situations. Even if the dataset is quite limited (19 participants), such a study in the context of a long-term postaccidental situation might be useful for radiation protection purposes, at least at the scale of a village, and for future dose assessment studies.

As a whole, the results of this project clearly demonstrate that even 30 years after the Chernobyl accident the population still has a strong interest in the radiological situation of their environment. Using this interest, this study brought together the inhabitants to discuss and educate around the issues of the quality of their environment. Thanks to the actions undertaken by the students, the awareness about the radiological situation in the village has substantially improved and their radiation protection culture and skills undoubtedly increased. However, the project also revealed that a continuous and regular sensitization about good practices is needed and remains crucial in order to maintain the vigilance within areas affected by nuclear accidents over the long term. The mechanisms to be used for maintaining such long term vigilance have to be studied and identified. A close and open-minded cooperation between experts, national and local authorities and the population living in contaminated areas appears to be the most viable and effective approach (Schneider et al., 2019).

\section{Acknowledgements}

The authors wish to warmly thank Ms Aliona Mikhailova for her expert assistance with the on-site logistic organization and huge translation work during the whole project. Many thanks also to the students and population who participated in the project and especially Ms. Galina Vasenovich, head of Education,
Sports and Tourism Department of the Bragin District Executive Committee, Mr. Fyodor Yermakov Director of the Komarin school, Mr. Vladimir Masalyka professor of physics at the Komarin school, Ms. Anastasia Fedosenko, former radiometrist for the Komarin village, and the group of 19 students implicated in the study: Polina Bliznet, Dmitry Goroshko, Denis Gudoshnikov, Karina Kartash, Oleg Kraisvetny, Rimma Krivonosova, Daniil Lyubich, Olga Panteleeva, Snezhana Pinchuk, Karina Povod, Philip Romanenko, Sophia Suprun, Wyacheslav Tarasov, Cyril Shapetko, Igor Shapetko, Vladimir Shiman, Wyacheslav Tarasov, Andrei Yakimovich, Julia Zhuravskaya.

TERRITORIES is part of the CONCERT project. This project has received funding from the Euratom research and training programme 2014-2018 under grant agreement No. 662287. This publication reflects only the author's view. Responsibility for the information and views expressed therein lies entirely with the authors. The European Commission is not responsible for any use that may be made of the information it contains.

\section{References}

Adachi $\mathrm{N}$ et al. 2016. Measurement and comparison of individual external doses of high-school students living in Japan, France, Poland and Belarus - the "D-shuttle" project. J. Radiol. Prot. 36: 49-66.

Ando R. 2018. Trust-what connects science to daily life. Health Phys. 115: 581-589.

Bataille C, Croüail P. 2005. Analyse des dispositifs réglementaires concernant le contrôle et le suivi de la contamination des sols, des denrées alimentaires et des produits commerciaux en Biélorussie. Fontenay aux roses: CEPN, p. 54 ( in French).

Bernhardsson C, Zvonova I, Raaf C, Mattsson S. 2011. Measurements of long-term external and internal radiation exposure of inhabitants of some villages of the Bryansk region of Russia after the Chernobyl accident. Sci. Total Environ. 409: 4811-4817.

Bottollier-Depois JF, Allain E, Baumont G, Berthelod N, Clairand I, Couvez C, Darley G, Henry B, Jolivet T, Laroche P, Lebeau-Live A, Lejeune V, Miss J, Monange W, Quéinnec F, Richet Y, Simon C, Trompier F, Vayron F. 2017. OPEN RADIATION: A collaborative project for radioactivity measurement in the environment by the public. Eur. Phys. J. Conf. 153: 08002.

Bottollier-Depois JF, Allain E, Baumont G, Berthelot N, Darley G, Ecrabet F, Jolivet T, Lebeau-Livé A, Lejeune V, Queinnec F, Simon C, Trompier F. 2019. The OpenRadiation project: Monitoring environmental radioactivity by and for the citizens. Radioprotection 54(4): 241-246

Brown A, Franken P, Bonner S, Dolezal N, Moross J. 2016. Safecast: Successful citizen-science for radiation measurement and communication after Fukushima. J. Radiol. Prot. 36: S82-S101.

Dubreuil GH, Lochard J, Girard P, Guyonnet JF, Le Cardinal G, Lepicard S, Livolsi P, Monroy M, Ollagnon H, Pena-Vega A, Pupin V, Rigby J, Rolevitch I, Schneider T. 1999. Chernobyl post-accident management: The ETHOS project. Health Phys. 77: 361-72.

Hirayama H. 2013. An evaluation of personal dosimeter for widely distributed ${ }^{134} \mathrm{Cs}$ and ${ }^{137} \mathrm{Cs}$ by using EGS code. Radioisotopes 62 : 335-345.

Hultquist C, Cervone G. 2018. Citizen monitoring during hazards: Validation of Fukushima radiation measurements. GeoJournal 83: 189-206.

ICRP. 2009. Application of the commission's recommendations to the protection of people living in long-term contaminated areas after a 
nuclear accident or a radiation emergency. ICRP Publication 111. Ann ICRP 39: 74.

ICRP. 2018. Ethical foundations of the system of radiological protection. ICRP Publication 138. Ann ICRP 47: 1-65.

IRSN. 2016. Exposition de la population française aux rayonnements ionisants. Fontenay-aux-Roses: IRSN, p. 23 (in French).

Liland A, Skuterud L. 2013. Social and ethical aspects of radiation risk management (D. Oughton, S.O. Hansson, Eds.) pp. 159-176. Amsterdam: Elsevier.

Lochard J. 2007. Rehabilitation of living conditions in territories contaminated by the Chernobyl accident: The ETHOS project. Health Phys. 93: 522-526.

Lochard J, Schneider T, Ando R, Niwa O, Clement C, Lecomte JF, Tada JI. 2019. An overview of the dialogue meetings initiated by ICRP in Japan after the Fukushima accident. Radioprotection 54 (2), 87-101. DOI: 10.1051/radiopro/2019021.

Miyazaki M. 2017. Using and explaining individual dosimetry data: Case Study of four municipalities in Fukushima. Asia Pac. J. Public Health 29: 110S-119S.
Nursal WI, Okuda T, Toshihiro Y. 2016. Potential of crowdsourcing approach on monitoring radioactivity in Fukushima prefecture. Glob. Environ. Res. 20: 111-118.

Reiher C. 2016. Lay people and experts in citizen science: Monitoring radioactively contaminated food in post-Fukushima Japan. Asien 140: $56-73$.

Schneider T, Maître M, Lochard J, Charron S, Lecomte J F, Ando R, Kanai Y, Kurihara M, Kuroda Y, Miyazaki M, Naito W, Orita M, Takamura N, Tanigawa K, Tsubokura M, Yasutaka T. 2019. The role of radiological protection experts in stakeholder involvement in the recovery phase of post nuclear accident situations: Some lessons from the Fukushima-Daïchi NPP accident. Radioprotection 54(4): 259-270

UNSCEAR. 2008. Annex B: Exposures of the public and workers from various sources of radiation. In: Sources and effects of ionizing radiation (UNSCEAR, Ed.). New-York: United Nations.

UNSCEAR. 2011. Annex D: Health effects due to radiation from the Chernobyl accident. In: Sources and effects of ionizing radiation (UNSCEAR, Ed.). New-York: United Nations.

Cite this article as: Bertho J-M, Maître M, Croüail P, Naito W, Shkliarava N, Mostovenko A, Jones K, Simon-Cornu M. 2019. Assessment of population radiation exposure at the edge of the exclusion zone 32 years after the Chernobyl accident: methods and preliminary results. Radioprotection 54(4): 247-257 